\title{
BMJ Open Results of a feasibility randomised clinical trial on pain education for low back pain in Nepal: the Pain Education in Nepal-Low Back Pain (PEN-LBP) feasibility trial
}

Saurab Sharma, ${ }^{\oplus 1,2}$ Mark P Jensen, ${ }^{3}$ G Lorimer Moseley, ${ }^{4}{\mathrm{~J} \mathrm{Haxby} \text { Abbott }^{\odot 2}}^{2}$

To cite: Sharma S, Jensen MP, Moseley GL, et al. Results of a feasibility randomised clinical trial on pain education for low back pain in Nepal: the Pain Education in NepalLow Back Pain (PEN-LBP) feasibility trial. BMJ Open 2019;9:e026874. doi:10.1136/ bmjopen-2018-026874

- Prepublication history and additional material for this paper are available online. To view these files, please visit the journal online (http://dx.doi. org/10.1136/bmjopen-2018026874).

Received 24 September 2018 Revised 15 January 2019 Accepted 6 February 2019
Check for updates

(C) Author(s) (or their employer(s)) 2019. Re-use permitted under CC BY-NC. No commercial re-use. See rights and permissions. Published by BMJ.

For numbered affiliations see end of article.

Correspondence to

Saurab Sharma;

saurabsharma1@gmail.com

\section{ABSTRACT}

Objectives The aims of this study were to: (1) develop pain education materials in Nepali and (2) determine the feasibility of conducting a randomised clinical trial (RCT) of a pain education intervention using these materials in Nepal.

Design A two-arm, parallel, assessor-blinded, feasibility RCT.

Setting A rehabilitation hospital in Kathmandu, Nepal. Participants Forty Nepalese with non-specific low back pain (mean [SD] age 41 [14] years; 12 [30\%] women). Interventions Eligible participants were randomised, by concealed, 1:1 allocation, to one of two groups: (1) a pain education intervention and (2) a guideline-based physiotherapy active control group intervention. Each intervention was delivered by a physiotherapist in a single, 1-hour, individualised treatment session.

Primary outcome measures The primary outcomes were related to feasibility: recruitment, retention and treatment adherence of participants, feasibility and blinding of outcome assessments, fidelity of treatment delivery, credibility of, and satisfaction with, treatment. Assessments were performed at baseline and at 1 week post-treatment.

Secondary outcome measures Pain intensity, pain interference, pain catastrophising, sleep disturbance, resilience, global rating of change, depression and quality of life. Statistical analyses were conducted blind to group allocation.

Results Forty participants were recruited. Thirtyeight participants (95\%) completed the 1-week post-treatment assessment. Most primary outcomes surpassed the a priori thresholds for feasibility. Several findings have important implications for designing a full trial. Secondary analyses suggest clinical benefit of pain education over the control intervention, with larger decrease in pain intensity (mean difference $=3.56$ [95\% Cl 0.21 to 6.91]) and pain catastrophising (mean difference $=6.16$ [95\% $\mathrm{Cl} 0.59$ to 11.72]) in the pain education group. Pain intensity would seem an appropriate outcome for a full clinical trial. One minor adverse event was reported.

Conclusion We conclude that a full RCT of pain education for back pain in Nepal is feasible and warranted.

Trial registration number NCT03387228; Results.
Strengths and limitations of this study

- This is the first study to examine the feasibility of a clinical trial on low back pain in Nepal.

- We developed a culturally suitable pain education programme using local patient stories before using it in the feasibility trial.

- We blinded the assessor and data analyst to group allocation; however, due to the nature of the intervention, we could not blind the therapists and study participants.

- We used guideline-based care as an active control group.

- Conclusions regarding the effectiveness of the intervention should not be made because this was a feasibility study, not a clinical trial; however, significant between-group differences on proposed outcome measures justify proceeding with a full definitive trial.

\section{INTRODUCTION}

Low back pain (LBP) is the leading cause of disability in both low-income and high-income countries and is associated with large direct (healthcare) and indirect costs. ${ }^{1-3}$ The limited available literature on LBP in Nepal indicates LBP prevalence of between $35 \%$ and $65 \%^{45}$ and that prevalence will probably increase in the next decade. ${ }^{3}$ Therefore, timely use of interventions that are evidence informed, effective and inexpensive is urgently required.

Internationally, clinical practice guidelines on LBP consistently recommend non-pharmacological and non-surgical approaches as the first line of treatment. ${ }^{6-8}$ For acute back pain, core common recommendations are education or advice for reassurance, remaining active, returning to work and avoiding bed rest and lumbar supports. For chronic back pain, recommendations are education, 
exercise and psychological therapies. ${ }^{6-9}$ Remarkably, although many high-income countries are moving away from primarily drug and surgical management of LBP because of their associated risks and costs, and general lack of efficacy, ${ }^{10}$ such interventions are now increasingly provided in Nepal. ${ }^{11}{ }^{12}$ Unfortunately, there is little or no research, nor clinical evidence, that evaluates the efficacy of any treatments for LBP in Nepal, including the firstline treatments that are now recommended in clinical guidelines elsewhere.

Although education is almost universally recommended for LBP, there are no clear curricula for delivering it, and little attention is given to training, methods, settings or context. ${ }^{13}$ One type of education that is an exception to this rule and has been widely studied focuses on improving patient understanding of the biological mechanisms that underpin pain and how best to promote recovery. ${ }^{14} 15$ This form of pain education (widely known as 'Explain Pain' or 'Pain Neuroscience Education') ${ }^{15-24}$ was developed in Australia and has been adapted in numerous Western countries, demonstrating mixed results for effectiveness for managing LBP. ${ }^{151721}$ 23-25 Education can be brief, around $10 \mathrm{~min}$ to deliver the key messages, or extended (1 hour to several hours). Although no strong evidence exists in support of effectiveness of longer versus shorter education duration, a short education session is time efficient. However, longer forms of pain education have several advantages over shorter forms; specifically, they allow for the integration of contemporary principles of conceptual change and education (eg, including stories and metaphors ${ }^{26}$ ) and can provide adequate time for guidance on self-management strategies such as graded exposure to difficult or painful activities. ${ }^{1527} 28$ Longer duration pain education also allows for greater tailoring of individual curriculum and target concepts, provides patients with time and opportunity to voice doubts and ask questions and allows the clinician to assess learning in real time..$^{28}$

Treatment that is effective in one culture may not necessarily be effective in another. We know of no reports of pain education being adapted or evaluated within an Eastern cultural context. The critical first step then is to determine whether indeed it is feasible to do so. ${ }^{29} \mathrm{We}$ therefore: (1) developed evidence-based pain education materials in Nepali for application in tertiary and primary care settings in Nepal and (2) investigated the feasibility of conducting a randomised clinical trial (RCT) comparing effectiveness of pain education to an appropriate control condition. We aimed to determine whether it would be feasible to undertake a full RCT within the Nepalese healthcare system and to identify any modifications that may be needed before doing so.

\section{METHODS}

The research was conducted in two stages. First, we developed pain education materials in Nepali, followed by a

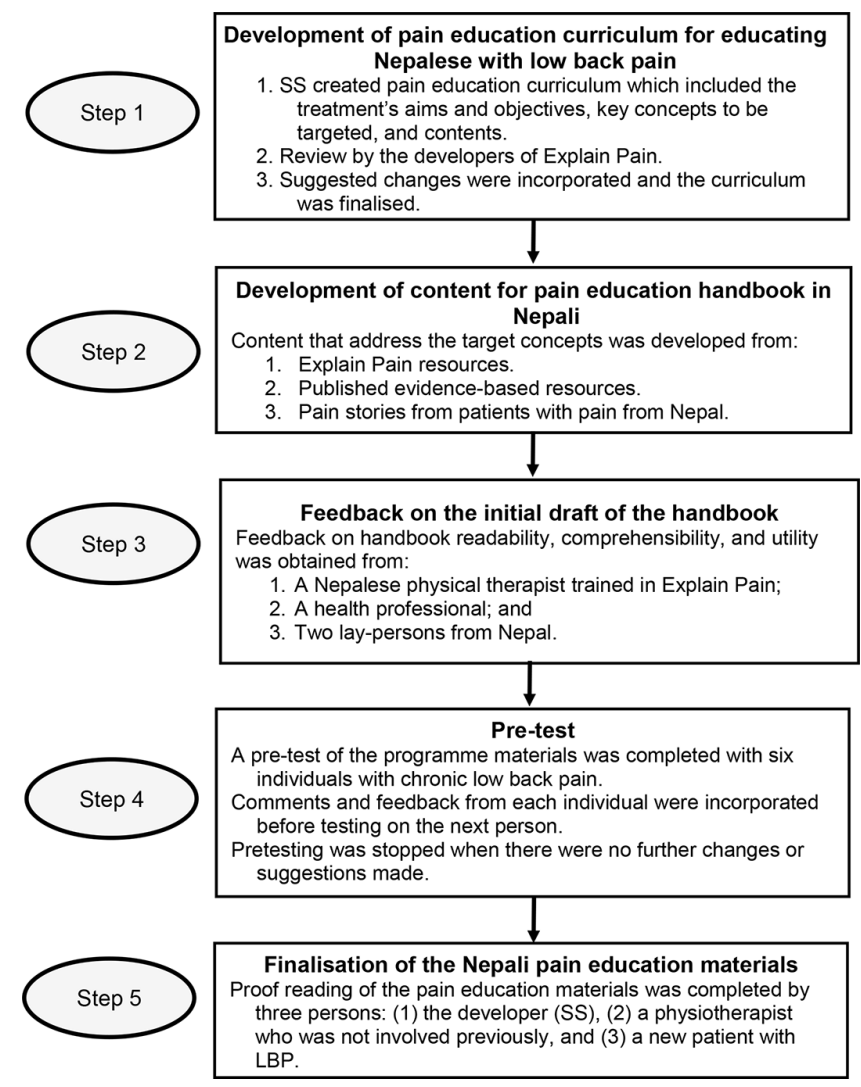

Figure 1 Steps in the development of pain education handbook. LBP, low back pain.

feasibility trial evaluating the feasibility of conducting an RCT to evaluate the effectiveness of pain education.

\section{Development of pain education in Nepali}

The primary investigator (SS) developed the pain education resources in Nepali, based on the 'Explain Pain' pain education materials (NOIgroup Publishing, Adelaide, Australia). ${ }^{28}{ }^{30}$ Figure 1 lists the development process, which included five steps.

In the first step, SS developed a context and culture-specific pain education curriculum according to the process set out in Moseley and Butler. ${ }^{28}$ The curriculum was reviewed by the authors of that guide (including coauthor of this paper: GLM). Four key concepts (described below) were identified, with one additional optional concept if time permitted. The final curriculum, including the key concepts to deliver, details of contents and methods of delivery were published in our protocol paper ${ }^{31}$ and are also presented in online supplementary file 1 .

In the second step, a pain education handbook was created using contents from Explain Pain ${ }^{28} 30$ and clinical practice guidelines on LBP. ${ }^{6-8}$ We used pain stories from Nepal to help explain the target concepts. ${ }^{31}$ We kept the Nepalese adaptations as simple as possible, so that patients with little to no formal education would understand them.

In the third step, the material was reviewed by four Nepalese with a medical $(n=2)$ or non-medical $(n=2)$ background and revised as a result. In the fourth step, we 
undertook initial pilot testing of the pain education handbook with six patients with chronic LBP. We focused here on its readability, the relevance of the stories and whether the new pictures created for the handbook delivered their intended meaning. The handbook text was revised, but no changes were made to the pictures. Finally, three native Nepali-speaking persons proof-read the handbook, and a final version was completed.

\section{Research design}

We conducted a two-arm, assessor-blinded, feasibility RCT. We registered the trial protocol at ClinicalTrials.gov. We used the Standard Protocol Items: Recommendations for Interventional Trials statement ${ }^{32}$ during the development of the protocol and followed the Consolidated Standards of Reporting Trials statement extension for a pilot and feasibility randomised trials ${ }^{33}$ for reporting. Feasibility was to be determined based on a priori criteria. ${ }^{31}$ For the detailed review of the research methods, we refer readers to the published protocol. ${ }^{31}$

\section{Participants}

We included adults (age 18 years or more) with non-specific LBP of any duration. We excluded patients with specific causes of LBP such as malignancy, fracture, infection or inflammatory arthritis identified from history or investigations. We also excluded pregnant women and patients presenting with the history of bladder and bowel incontinence or perineal anaesthesia.

We recruited participants from a rehabilitation hospital in Kathmandu, Nepal. We invited consecutive patients presenting at the centre to participate in the study. Additionally, we made advertisements on social media about the research to improve the recruitment, as almost $28 \%$ of Nepalese use Facebook (www.internetworldstats.com). We provided an appointment to interested candidates for screening at the centre. A research assistant (a trained physiotherapist) screened all potential participants for eligibility who expressed a willingness to participate in the current study. All participants signed the consent form prior to baseline assessment.

\section{Interventions}

We used the Template for Intervention Description and Replication (TIDieR) Checklist to plan and report the study interventions. ${ }^{34}{ }^{35}$ There were two interventions in a two-arm RCT design. We provided pain education to the participants who were randomly allocated to the experimental group (pain education group [PEG]) and guideline-based physiotherapy treatment to the participants who were randomly allocated to the control group (CG). Treatment time for both groups was 1 hour.

\section{The PEG group: delivery of pain education}

The principal investigator (SS), who has received extensive training in Explain Pain via NOIgroup Professional Development and one-on-one mentoring with pain education experts, delivered the treatment. The pain education deliverer first asked two questions to the patients in the PEG: (1)
'Is there anything in particular that you would like to learn about your low back pain, or pain in general?' and (2) 'Do you know what caused your low back pain? Can you please explain the cause of your low back pain from what you have understood, or what you have been told?. Up to $15 \mathrm{~min}$ was allotted to addressing, with evidence-informed answers, any questions participants had and to clarify any misconceptions the patients had regarding their LBP. The rest of the session was used to deliver information regarding the target concepts.

\section{Target concepts delivered}

The key target concepts were: (1) pain is normal and almost everyone experiences it at different times during their life; (2) the body sends danger signals (ie, not necessarily information about physical damage, but the danger of potential physical damage), and the brain decides whether to produce pain; (3) learning about pain physiology changes pain, and anything previously associated with pain (eg, past learning, social factors and environmental cues) can influence current pain; and (4) the body can learn to experience pain and become more overprotective over time. One additional target concept "pain and tissue damage are poorly related' was delivered if there was time available after the four key concepts were addressed. During the pain education session, strategies for graded exposure to painful or difficult activities were also provided to the patients to increase their physical activity.

\section{Guideline-based physiotherapy treatment}

CG treatment consisted of guideline-based physiotherapy interventions extracted from recent clinical practice guidelines on LBP. ${ }^{6}{ }^{36}$ Criteria for the CG treatment component required that it be: (1) a first-line recommended treatment, or (2) a second-line recommended treatment to make the total duration of the session be 1 hour (to match PEG treatment time); (3) feasible to be delivered during the first clinical contact; and (4) one that is routinely delivered in, and can be competently delivered by, physiotherapists at the recruitment centre. Given these criteria, the CG treatment condition included: (1) brief education to reassure the patient, advice to remain active and remain at or return to work (if the participant had been working prior to pain onset), general education about the favourable prognosis of LBP that it will generally get better in two to 6 weeks and advice to avoid bed rest and lumbar corsets $(10-15 \mathrm{~min}),{ }^{6} 3637$ (2) superficial heat $(10-15 \mathrm{~min}),{ }^{637}$ (3) back massage $(10 \mathrm{~min})^{636}$ and (4) static cycling to promote physical activity (remaining time; between $20 \mathrm{~min}$ and 30 min). ${ }^{636}$ Although treatment in CG involved communication between the treating therapist and patients, this communication was strictly limited to providing either: (1) brief education as described above or (2) active listening. Key concepts delivered in the PEG were not provided to the patients in this group.

\section{Home treatment}

We also prescribed a home programme for both groups. This included a leaflet providing brief education on 
self-management of LBP, with pictures to remind the participants to remain physically active, education regarding positive prognosis, advice to walk for $30 \mathrm{~min}$ daily (with rest if required) and to avoid bed rest or lumbar corsets.

In addition to the leaflet that was also provided to the CG, participants in the PEG received the pain education handbook. We suggested to participants that they read the booklet at least once during the following week. If the patients could not read, they were advised to request a family member to read the pain education handbook to them. Adherence to both exercise (eg, walking) and reading the pain education handbook at home was recorded, by self-report, 1 week post-treatment.

Participants in both treatment groups were required to pay the same fee for physiotherapy services as usual for non-trial patients. This payment was identical for both interventions.

\section{Outcome measures}

Demographic data were collected as per the recommendations of the National Institutes of Health (NIH) task force on research standards for chronic LBP. ${ }^{38}$

\section{Primary outcome measures}

The primary outcomes were related to feasibility: recruitment, retention and treatment adherence of participants, feasibility and blinding of outcome assessments, fidelity of treatment delivery and credibility of, and satisfaction with, treatment. To assess recruitment-related feasibility outcomes, we recorded the numbers of potential participants who were eligible and recruitment rates. Participation-related feasibility outcomes were: (1) rates of willingness to participate in an RCT and (2) acceptability of random allocation to a treatment group. Feasibility outcomes related to outcome assessment were: (1) feasibility of assessor blinding procedures and (2) acceptability of screening procedures. Finally, the treatment-related feasibility outcomes were: (1) possible contamination between the groups, (2) the credibility and acceptability of the interventions, (3) adherence to the interventions, (4) treatment satisfaction, (5) difficulty in understanding the treatment and (6) adverse events related to the interventions. Details of these feasibility outcome measures are presented in online supplementary file 2 .

\section{Secondary outcome measures}

The secondary outcome measures selected were those that had the potential to be primary or secondary outcomes of a potential full clinical trial, based on the core outcome sets recommended for LBP. ${ }^{39}{ }^{40}$ We used eight outcome measures previously translated and cross-culturally adapted to the Nepali language: four Patient-Reported Outcome Measurement Information System (PROMIS) short form measures assessing pain intensity, pain interference, sleep disturbance and depression $^{41}$; a two-item quality of life scale, seven-point Global Rating of Change ${ }^{42}$; the Pain Catastrophizing
Scale $(\mathrm{PCS})^{44}$; and the 10 -item Connor Davidson Resilience Scale (CDRISC). ${ }^{45}$

\section{Sample size}

Sample size estimation was performed to achieve the primary feasibility outcomes goals, as described in the protocol $^{31}$ and registration documents, and not to detect differences in the secondary treatment effects outcomes. ${ }^{46}$ Based on guidance in the literature, ${ }^{47}$ the research team estimated that a sample size of 40 (20 in each treatment arm) would be sufficient to adequately evaluate the feasibility of undertaking a full clinical trial. $^{31}$

\section{Randomisation}

The published research protocol ${ }^{31}$ was strictly followed. Allocation sequence was generated in random blocks of 4 and 6 using www.random.org by a researcher (JHA) who was not involved in recruitment. Allocation concealment was performed using sequentially numbered opaque, sealed envelopes, prepared by JHA, and maintained until the interventions were assigned to the study participants. The group allocation was revealed to the study participants and intervention providers only after completion of the baseline assessment.

\section{Blinding}

The assessor performing all the assessments was blinded to group allocation of the participants throughout the study. The data analyst (SS) was also blinded to group allocation. That is, after the assessor entered data in the Excel spreadsheet without knowledge of group allocation, the entered data were sent to JHA, who added codes for group allocation (red and blue) before the data analyses were performed. Unblinding of group allocation occurred after all planned analyses were complete.

\section{Statistical methods}

Baseline characteristics for demographic and clinical data of the participants were reported using descriptive statistics. The plans for analysis of primary outcome measures are presented in online supplementary file 2 .

We planned the exploratory analysis of betweengroup differences in the secondary outcome measures using two-group t-tests, with the understanding that the current study was not powered to detect statistically significant between-group differences in the secondary outcomes. Rather, analyses of between group differences were computed primarily for descriptive purposes in order to inform decisions regarding the selection of measures for a possible future full clinical trial. The scores of the PROMIS measures were transferred into the template provided by www.assessmentcenter. net, which computed the total raw scores, T-scores and SEs. The assessment centre automatically handles missing items when performing the analysis. For other measures, missing items were imputed using the mean of the present items for that patient. The details of the 
measures with the psychometric properties are outlined in online supplementary file 3 .

\section{Patient and public involvement}

Patients with LBP and non-clinician volunteers provided significant feedback in the development of the Nepalese pain education materials. We incorporated real but anonymous pain-related stories of Nepalese so that the intervention is relatable. Neither patients nor members of the public were involved in the design of the study.

\section{RESULTS}

Data were collected between February and April 2018, with mean (SD; range) duration to follow-up of 7.63 (1.08; 7-11) days. Recruitment was stopped after achieving the desired sample size of 40 . Twenty participants were randomised to each treatment arm.

\section{Sample characteristics}

The majority of participants in each group were men, married and Hindu. Baseline demographic characteristics were comparable between the groups. However, baseline scores on the secondary outcomes were somewhat higher in the PEG than the CG. Details of the baseline sample characteristics are presented in table 1 .

\section{Missing data}

One item (item \#10) in the baseline assessment of the PCS and one item in the follow-up assessment of CDRISC (item \#8) were missing for one participant. Missing values were replaced by the mean score of the items responded to of each measure for that participant. One item in the baseline depression scale was missing for one participant, which was imputed by the PROMIS assessment centre during the analysis.

\section{Primary (feasibility) outcomes}

Results related to feasibility outcomes are presented in table 2, and summary results on feasibility criteria are presented in table 3 .

\section{Recruitment-related feasibility outcomes}

Seventy candidates were invited to participate in the study. Twenty-eight participants $(70 \%)$ were recruited from the data collection centre; $12(30 \%)$ from community advertisements. Fifty-seven per cent of invited candidates participated. Of those who did not, 27 (90\%) declined participation and $3(10 \%)$ did not meet inclusion criteria. Forty out of 43 candidates (93\%) screened were eligible to participate. All 40 participants $(100 \%)$ who met the inclusion criteria provided written informed consent and were randomised to one of the study arms. One participant in each group was lost to follow-up. The reasons for all exclusions and losses to follow-up are outlined in the participant flow diagram (figure 2).
Table 1 Baseline characteristics of the two study groups

\begin{tabular}{|c|c|c|}
\hline \multirow[b]{3}{*}{ Variable } & PEG & CG \\
\hline & $(n=20)$ & $(n=20)$ \\
\hline & $\begin{array}{l}\mathbf{N}(\%) \text { or } \\
\text { mean (SD) }\end{array}$ & $\begin{array}{l}\mathbf{N}(\%) \text { or } \\
\text { mean (SD) }\end{array}$ \\
\hline \multicolumn{3}{|l|}{ Recruitment, N (\%) } \\
\hline Advertisement & $6(30)$ & $6(30)$ \\
\hline Hospital & $14(70)$ & $14(70)$ \\
\hline \multicolumn{3}{|l|}{ Sex, N (\%) } \\
\hline Men & $15(75)$ & $13(65)$ \\
\hline Women & $5(25)$ & 7 (35) \\
\hline \multicolumn{3}{|l|}{ Marital status } \\
\hline Married & $16(80)$ & $15(75)$ \\
\hline Single & $4(20)$ & $3(15)$ \\
\hline Separated or widowed & $0(0)$ & $2(10)$ \\
\hline \multicolumn{3}{|l|}{ Religion, N (\%) } \\
\hline Hindu & $19(95)$ & $16(80)$ \\
\hline Buddhist & $1(5)$ & $3(15)$ \\
\hline Others & $0(0)$ & $1(5)$ \\
\hline \multicolumn{3}{|l|}{ Race/ethnicity, N (\%) } \\
\hline Chettri & $6(30)$ & $5(25)$ \\
\hline Brahmin & $4(20)$ & $9(45)$ \\
\hline Newar & $4(20)$ & $2(10)$ \\
\hline Others & $6(30)$ & $4(20)$ \\
\hline \multicolumn{3}{|l|}{ Education, N (\%) } \\
\hline No school & $3(15)$ & $2(10)$ \\
\hline Primary school (<5years) & $3(15)$ & $1(5)$ \\
\hline $\begin{array}{l}\text { Upto high school (6- } \\
12 \text { years) }\end{array}$ & $5(25)$ & $8(40)$ \\
\hline Bachelor degree and over & $9(45)$ & $9(45)$ \\
\hline \multicolumn{3}{|l|}{ Primary occupation, N (\%) } \\
\hline Business or office work & $13(65)$ & 7 (35) \\
\hline Unemployed & $0(0)$ & $5(25)$ \\
\hline Homemaker & $2(10)$ & $3(15)$ \\
\hline $\begin{array}{l}\text { Currently sick leave for } \\
\text { LBP }\end{array}$ & $1(5)$ & $1(5)$ \\
\hline Other & $4(20)$ & $2(10)$ \\
\hline \multicolumn{3}{|l|}{ Smoking history, N (\%) } \\
\hline Never smoked & $10(50)$ & $12(60)$ \\
\hline Currently smoker & $8(40)$ & $5(25)$ \\
\hline Have quit smoking & $2(10)$ & $3(15)$ \\
\hline \multicolumn{3}{|l|}{$\begin{array}{l}\text { Have left work for more } \\
\text { than } 1 \text { month due to LBP, } \\
\mathrm{N}(\%)\end{array}$} \\
\hline Yes & $4(20)$ & $4(20)$ \\
\hline No & $16(80)$ & $16(80)$ \\
\hline $\begin{array}{l}\text { Medications used for LBP, } \\
N(\%)\end{array}$ & & \\
\hline
\end{tabular}

Continued 
Table 1 Continued

\begin{tabular}{|c|c|c|}
\hline \multirow[b]{3}{*}{ Variable } & PEG & CG \\
\hline & $(n=20)$ & $(n=20)$ \\
\hline & $\begin{array}{l}\mathrm{N}(\%) \text { or } \\
\text { mean (SD) }\end{array}$ & $\begin{array}{l}\mathrm{N}(\%) \text { or } \\
\text { mean (SD) }\end{array}$ \\
\hline NSAIDs & $3(15)$ & $6(30)$ \\
\hline Pregabalin & $2(10)$ & $3(15)$ \\
\hline Vitamin $B_{12}$ & $3(15)$ & $1(5)$ \\
\hline Gabapentin & $1(5)$ & $0(0)$ \\
\hline Opioids & $1(5)$ & $0(0)$ \\
\hline Antidepressant & $1(5)$ & $0(0)$ \\
\hline \multicolumn{3}{|l|}{ Secondary outcomes } \\
\hline Pain intensity ${ }^{*}$ & $54.38(3.48)$ & $52.72(2.45)$ \\
\hline Pain interference ${ }^{\star}$ & 62.28 (6.62) & $58.92(7.69)$ \\
\hline Sleep disturbance* & $51.84(7.68)$ & $45.63(8.71)$ \\
\hline Depression* & $56.99(8.08)$ & 53.60 (11.25) \\
\hline Quality of life & $5.70(1.22)$ & $6.10(1.21)$ \\
\hline Pain catastrophising & $22.70(10.99)$ & 20.50 (12.56) \\
\hline Resilience & $26.95(9.14)$ & $28.60(8.08)$ \\
\hline
\end{tabular}

${ }^{\star} T$ scores.

CG, control group; LBP, low back pain; NSAIDs, non-steroidal antiinflammatory drugs; PEG, pain education group.

\section{Participant-related feasibility outcomes}

\section{Willingness to participate in a randomised trial}

The main reasons for unwillingness to participate were: (1) wanting to receive comprehensive physiotherapy treatment as an in-patient $(\mathrm{n}=8),(2)$ not wanting to pay for treatment $(\mathrm{n}=6)$, (3) not having time to participate in the study and complete the post-treatment assessment at 1 week $(n=6)$ and (4) wanting to receive electrotherapy treatment for 1 week because it was recommended by their physician $(n=5)$.

\section{Acceptability of random allocation to a treatment group}

Random allocation of the treatment was acceptable to 57 out of 70 individuals $(81 \%)$. Of the 13 participants who did not accept random allocation, $5(7 \%)$ wanted to receive electrotherapy treatment specifically and $8(11 \%)$ wanted to be admitted at the centre to receive comprehensive physiotherapy treatment (including electrotherapy) twice a day for a week as advised by their treating physician or physiotherapists.

\section{Outcomes assessment-related feasibility outcomes Feasibility of blinding the assessor}

The assessor did not receive any definitive information about participants' group allocation for any of the participants during the study. The assessor's guess was correct for 12 participants $(60 \%)$ in the PEG condition and for 11 participants (55\%) in the CG condition. On questioning, the assessor identified some clues that may have influenced a correct guess: (1) 'duration of treatment time' (see below) ( $\mathrm{n}=5$; three correct and two incorrect guesses), (2) patients reporting the treatment as 'interesting' $(\mathrm{n}=2$; both incorrect guesses) and (3) the treating therapist's description of the treatment as interactive ( $\mathrm{n}=1$; correct guess).

\section{Acceptability of screening procedures by the assessor}

Mean (SD; range) time taken to complete the screening process (including time to sign the consent) was 7 (6; 6-45) minutes. Mean (SD; range) time taken to complete all the forms during the baseline assessment was 20 (5; 12-35) minutes.

The screener reported that the screening procedures were acceptable, but there were two problems. First, the duration of screening was occasionally too long, for example, when patients told stories about their pain rather than keeping answers focused on the questions that were asked, or an accompanying friend kept responding on the patient's behalf. Second, interspersing assessments unrelated to pain (eg, CDRISC, sleep disturbance, depression and quality of life) between assessments related to pain (eg, pain intensity and pain interference) made it difficult for some participants to switch focus between the pain and general domains. As a result, some participants kept answering about pain when the questions asked about other domains such as sleep or depression.

\section{Treatment-related feasibility outcomes}

\section{Contamination}

There were no detected instances of contamination between the two groups. Table 2 presents the results of the five separate contamination questions.

\section{Credibility and acceptability of the interventions}

The credibility scores of the two conditions at 1-week assessment and average treatment time were similar (table 2). Both interventions were acceptable to all the participants. However, patients in the PEG often expected some form of physiotherapy interventions in addition to education. For example, one patient, assigned to the PEG condition, had severe pain and stated that he wanted a physical treatment for his back pain. Similarly, most of the patients in the CG mostly expected back-specific exercises and/or electrotherapy treatment over the painful sites. One comment from a participant after completing cycling was 'Okay, this was exercise for my general health. What exercise should I perform for my back pain?'. Similarly, many participants in the CG were keen to receive pain education intervention, which they did $(n=15)$ after post-treatment assessment at 1 week.

\section{Adherence to intervention and treatment satisfaction}

Adherence to intervention and treatment satisfaction were similar in both groups (table 2). Twelve out of 38 patients who completed the post-treatment assessment at 1 week (32\%; five in the PEG and seven in the CG) wished to receive their regular physiotherapy treatment (mostly electrotherapy) at the centre between the two assessment time-points; these participants did receive this treatment as requested. 
Table 2 Feasibility results for the two study groups

\begin{tabular}{|c|c|c|c|c|}
\hline Feasibility outcomes & $\begin{array}{l}\text { PEG } \\
\text { N (\%) or } \\
\text { Mean (SD) }\end{array}$ & $\begin{array}{l}\text { CG } \\
\text { N (\%) or } \\
\text { Mean (SD) }\end{array}$ & $\begin{array}{l}\text { Mean difference } \\
(95 \% \mathrm{Cl}) \text { or } p \text { values }\end{array}$ & Summary \\
\hline Attrition rate & $1(5 \%)$ & $1(5 \%)$ & 1.000 & $\begin{array}{l}\text { No difference in attrition rates between } \\
\text { groups. }\end{array}$ \\
\hline $\begin{array}{l}\text { Assessor's correct guess for group } \\
\text { allocation }\end{array}$ & $12(60 \%)$ & $11(55 \%)$ & 0.756 & $\begin{array}{l}\text { Assessor correctly guessed the group } \\
\text { allocation slightly more often for the } \\
\text { PEG than the CG. }\end{array}$ \\
\hline $\begin{array}{l}\text { 1. Have you talked to other } \\
\text { participants about the intervention? }\end{array}$ & $0(0 \%)$ & $0(0 \%)$ & - & \\
\hline $\begin{array}{l}\text { 2. If yes, was your attitude/ } \\
\text { intervention changed? }\end{array}$ & $0(0 \%)$ & $0(0 \%)$ & - & \\
\hline $\begin{array}{l}\text { 4. Are participants in the other group } \\
\text { aware of the type of intervention you } \\
\text { are receiving? }\end{array}$ & $0(0 \%)$ & $0(0 \%)$ & - & \\
\hline $\begin{array}{l}\text { 5. For the control group: did you } \\
\text { read the pain education booklet } \\
\text { provided to the experimental group? }\end{array}$ & - & $0(\%)$ & - & \\
\hline
\end{tabular}

Credibility and acceptability of

interventions (scale 0-20)

Baseline assessment $(n=20)$

Final assessment $(n=19)$

Adherence to treatment

(number of days)

Followed advice $(n=19)$

Performed home exercises (mean days [SD])

$\begin{array}{lll}12.55(2.89) & 12.95(3.80) & 0.40(-2.56 \text { to } 1.76) \\ 12.37(2.63) & 12.26(4.17) & 0.11(-2.19 \text { to } 2.40)\end{array}$

Similar credibility scores between groups.

Participants were adherent to the treatment in both groups, with significantly more adherence reported by the CG participants.

Slightly more CG participants received regular physiotherapy at the centre, massage or acupuncture, and NSAIDs.

$\begin{array}{llll}\begin{array}{l}\text { Number of patients who received other } \\ \text { treatments (total) }\end{array} & 7(37 \%) & 0.471 \\ \text { Regular physiotherapy at the centre* } & 4(21 \%) & 5(26 \%) & 0.719 \\ \text { Massage or acupuncture } & 1(5 \%) & 2(10 \%) & 0.563 \\ \text { Number of NSAIDs per week used at } 2 & 5 & -3\end{array}$

follow-up

\begin{tabular}{|c|c|c|c|c|}
\hline $\begin{array}{l}\text { Total treatment time } \\
\text { (in minutes) }\end{array}$ & $61.00(7.88)$ & $60.60(8.85)$ & & $\begin{array}{l}\text { Treatment time is very similar between } \\
\text { the two treatment conditions and } \\
\text { consistent with the planned treatment } \\
\text { duration of treatment. }\end{array}$ \\
\hline Difficulty (scale 0-5); mean (SD) & $2.26(0.56)$ & $2.16(0.60)$ & \multirow[t]{5}{*}{$0.10(-0.28$ to 0.49$)$} & \multirow{5}{*}{$\begin{array}{l}\text { Majority of the participants }(75 \%) \\
\text { reported both treatments as easy. There } \\
\text { were no significant between-group } \\
\text { difference in difficulty score. }\end{array}$} \\
\hline Easy & $15(75 \%)$ & $15(75 \%)$ & & \\
\hline Neither easy nor difficult & $3(15 \%)$ & $2(10 \%)$ & & \\
\hline Difficult & $1(5 \%)$ & $1(5 \%)$ & & \\
\hline Very difficult & $0(0 \%)$ & $0(0 \%)$ & & \\
\hline
\end{tabular}

*Mostly included electrotherapy treatment.

CG, control group; NSAIDS, non-steroidal anti-inflammatory drugs; PEG, pain education group. 
Table 3 Were the feasibility criteria met?

\begin{tabular}{|c|c|c|}
\hline Criteria & $\begin{array}{l}\text { Feasibility } \\
\text { criteria met? }\end{array}$ & Recommendations for full trial \\
\hline Blinding of assessor & Yes & $\begin{array}{l}\text { Treatment providers should try to keep the treatment duration close to or equal to } \\
1 \text { hour to avoid any guesses of group allocation between the treatment groups. }\end{array}$ \\
\hline Recruitment rate & Yes & $\begin{array}{l}\text { Incorporating advertisement to recruit the patients was a good idea, which should } \\
\text { be considered in the full trial. }\end{array}$ \\
\hline Attrition rate (in both arms) & Yes & $\begin{array}{l}\text { Phone call reminders for the follow-up assessment helped reduce the drop-outs and } \\
\text { which should be considered in the future trial. }\end{array}$ \\
\hline Credibility of treatment & Yes & $\begin{array}{l}\text { The credibility scores of the two treatment conditions were within } 0.50 \mathrm{SD} \text { of each } \\
\text { other; therefore, no changes in the treatment conditions are required. }\end{array}$ \\
\hline Adherence to treatment & Yes & $\begin{array}{l}\text { Not many patients read the handbook provided to them. Creating interesting short } \\
\text { audios or videos with the key messages may be helpful for improving the adherence } \\
\text { to home advice. }\end{array}$ \\
\hline Difficulty level of the intervention & No & $\begin{array}{l}\text { A large proportion of patients reported the interventions to be 'easy'. The complexity } \\
\text { of the pain education content may be increased by providing more complex } \\
\text { neurophysiological knowledge to the patients. However, this may demand longer } \\
\text { duration of treatment time, and/or compromise the effectiveness of the intervention, } \\
\text { and may require pretesting of the changed intervention before using it in the full trial. }\end{array}$ \\
\hline
\end{tabular}

\section{Difficulty in understanding the treatment}

In both groups, 15 participants $(75 \%)$ reported that the treatment was 'easy' to understand (table 2). This result contravened our a priori cut-off point for this criterion of $50 \%$.

\section{Adverse events}

One participant in the CG reported lower extremity pain after cycling for $20 \mathrm{~min}$. The increase in her lower extremity pain lasted for 2 days and then subsided. None of the other participants reported any other adverse events associated with the treatments.

\section{Results of secondary outcomes}

We found significant within-group improvements from pre-treatment to post-treatment in all the secondary outcomes, except resilience for the PEG participants. In the CG group, we found pre-treatment to post-treatment improvements in pain interference, depression and catastrophising. We found statistically significant betweengroup differences in favour of PEG for pain intensity and pain catastrophising (table 4).

\section{Other findings}

The standard LBP treatment protocol at the data collection centre typically included non-guideline-based care such as advice to rest, advice against physical activity, admission for bed rest and intensive passive therapies (mostly electrotherapy). Such a care pathway contrasts with the recommendations and treatments presented in both groups. We found it challenging to alter the physiotherapists' usual practice.

Related to this, all of the physiotherapists who provided the CG treatment reported being dissatisfied with not being able to provide interventions they would normally provide, many of which were treatments that patients also wanted to receive, such as spine-specific exercises and manual therapies. Moreover, five of the physiotherapists who were initially trained in the guideline-based care prior to the initiation of the study left the treatment centre during the trial recruitment period. They were replaced by four physiotherapists who therefore had not been trained in guideline-based care as part of this study.

\section{DISCUSSION}

We aimed to determine whether it would be feasible to undertake a full RCT within the Nepalese healthcare system and to identify any modifications that may be needed before doing so. Seven of the eight a priori feasibility criteria were met, which suggests that a clinical trial to evaluate the effectiveness of pain education and evidence-based physiotherapy treatment in Nepal is feasible. This feasibility trial also provided important additional information that inform the design of the full trial. 


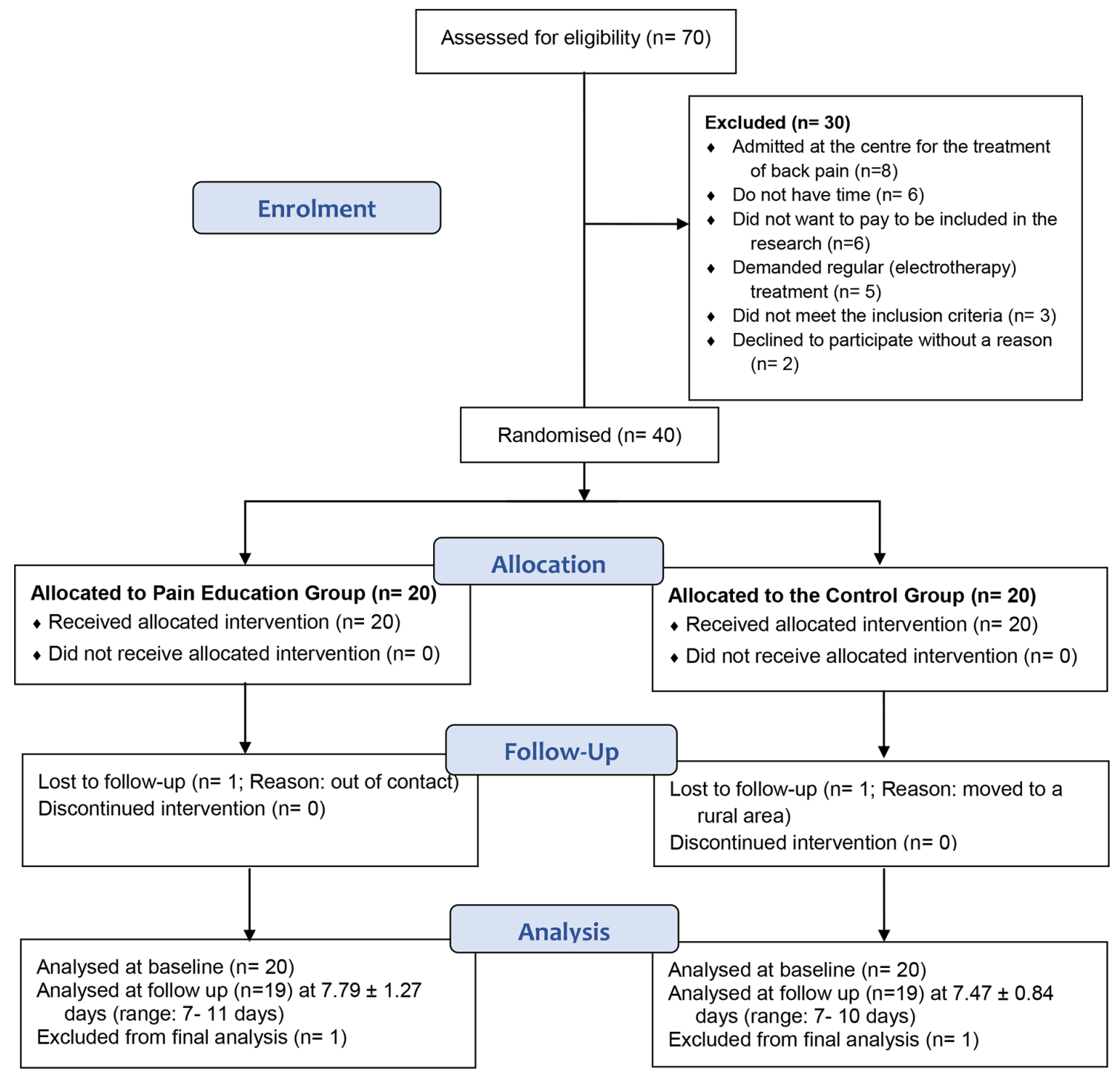

Figure 2 Participant flow diagram.

\section{Primary feasibility outcomes}

The recruitment rate exceeded our target of four participants enrolled every week. We used advertisements in social media, and we suspect that recruitment was aided by patient-to-patient word of mouth as the trial progressed. This, and the finding that our attrition rate (5\%) was well below our a priori maximum rate of $20 \%$ (which is thought to lead to serious threats to validity ${ }^{48}$ ) was surprising, considering that most patients in both groups did not receive the care they expected to receive. This is encouraging because it suggests that a broader education strategy, to prepare potential patients for an alternative approach to their problem before including them in a trial, is probably not required.

Although screening and data collection procedures were generally acceptable to the assessor, the assessor provided important recommendations to improve overall screening and data collection. For example, extended assessment sessions might be avoided by upskilling the assessors in dealing with patients, who are often elderly and uneducated and who tend to tell stories about their pain rather than provide direct answers to the questions being asked. An important caveat here, however, is the potentially critical role that this extra time and attentionparticularly insofar as it is dedicated to listening to patient stories-may have had in subsequent engagement and participation, particularly against the backdrop of unexpected care. The patients' stories in fact provide a context and meaning of their health problems, ${ }^{49}$ which may be a therapeutic intervention in itself, and are important to establish a good doctor-patient relationship. ${ }^{50}$ Clearly, the cost-benefit relationship of time-limited assessment is likely to be individually specific and nuanced.

The advantages and disadvantages of interviewing patients without their friends or family members present are also worthy of consideration. There were instances when excluding an accompanying family member would have reduced the data collection time and possibly improved the accuracy of the answers. However, the relationships patients have with those around them play an important role in the experience of pain ${ }^{51} 52$ and what people do about $\mathrm{it}^{51}$; exclusion of important others at a 


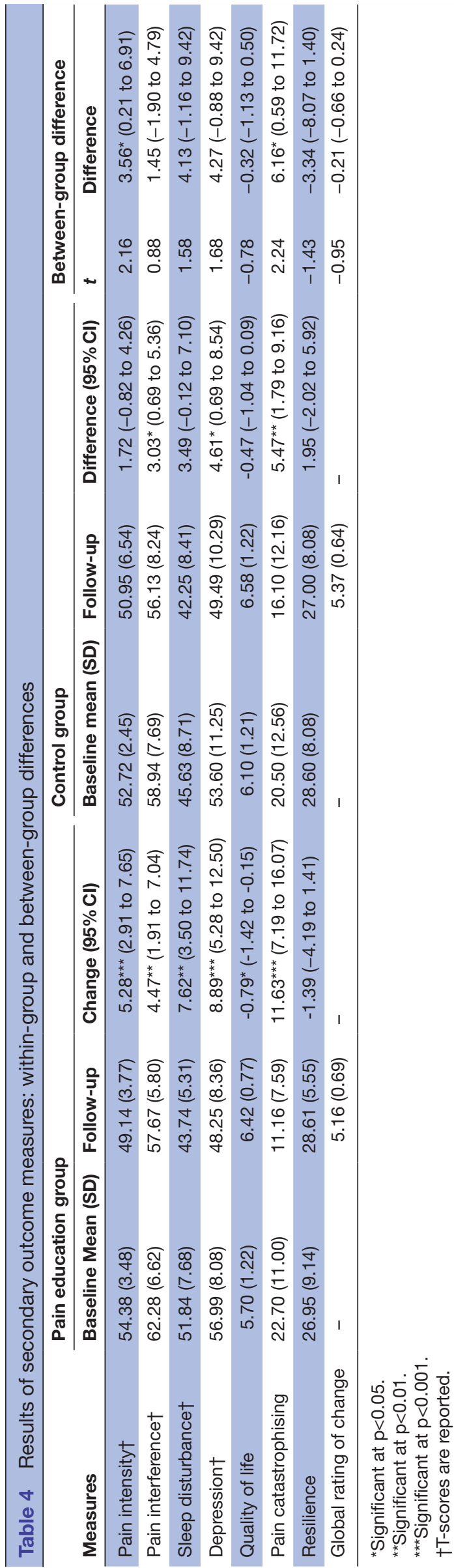

critical time may also disengage the patient or instil other barriers to their participation in the project. A final pragmatic modification to improve assessment would be to organise pain-related and pain-unrelated questions into different sections of the data collection protocol, so as to avoid patients being confused regarding the domains being assessed.

Blinding appeared to be successful, and contamination appeared to be avoided. Most controlled trials do not adequately examine assessor blinding, ${ }^{53}$ even though it is widely considered a very important component of good study design. ${ }^{54}$ We were able to blind the assessor here because we could provide a separate office space that was isolated from the treatment area. We were also able to schedule appointments to avoid contact with assessors that would unblind them to group assignment. Our inclusion of participant-reported items to evaluate contamination is not routinely included in feasibility or full clinical trials; the common approach is to implement strategies to minimise the risk a priori but not investigate it post hoc. However, in settings such as that involved here, where the community is well connected and word of mouth appears to be a significant recruitment pathway, we considered it important to also examine potential contamination post hoc. Limiting the number of patient recruitments performed in a single day to two may also have helped avoid contamination, but to see no evidence of contamination was surprising.

Treatment credibility and satisfaction were high for both groups (even though the participants did not receive the treatment they expected). That most participants in the PEG found the material 'easy' or 'very easy' was surprising and contrary to an a priori feasibility criterion. Our protocol ${ }^{31}$ stipulated a response to this outcome requiring that the material presented be viewed as difficult before proceeding to full trial. Whether we should increase difficulty by increasing the number of concepts covered, or going more fully into the four concepts we chose, or both, will require some pilot testing. That secondary outcome data findings suggesting a beneficial effect in the PEG condition appears to support making such a change.

\section{Secondary outcomes}

Statistically significant improvements over time were observed for $7 / 8$ outcomes in the PEG condition and 4/8 outcomes in the CG condition. Although assessment of the effectiveness of the interventions on the secondary outcomes was not a primary aim of this study, the significant between-group differences that were found were in favour of PEG, and the apparent effect was substantial on two key target outcomes: pain intensity and pain catastrophising (table 4). However, the consistently larger improvements in all of the other outcomes for the PEG condition, relative to the CG condition, suggests the possibility of wide benefits of pain education as compared with guideline care in Nepal. It should be remembered that these are secondary outcomes, not corrected for multiple 
analyses and therefore at risk of false positive results. However, these results add pertinence to the feasibility results; a full-scale clinical trial appears warranted.

\section{Recommendations}

Although the findings suggest that a clinical trial evaluating effectiveness of an adapted pain education intervention within Nepali primary and tertiary care is feasible, some improvements could be made. First, to improve the compliance of the physiotherapists with the CG treatment-guideline-based care-and adherence of patients to that care, the CG treatment condition may need to be modified. Ideally, this modification would be made so that the control treatment was consistent with the evidencebased practice paradigm as much as possible, for example, by giving participating patients and their therapists the ability to choose treatments that are mostly consistent with guideline recommendations for LBP treatment. For example, the guideline-based treatment could have two components: (1) mandatory first-line care recommended by the guidelines (education and reassurance, promotion of physical activity, early return to work and advice about positive prognosis for back pain), in addition to (2) a more pragmatic approach to LBP treatment. This second component may include any form of exercise (treadmill, static cycling or back-specific motor control or movement exercises), manual therapy (massage, mobilisation or manipulation, based on therapist's preference) or electrotherapy treatment, according to therapist and patient preference (as per recommendations of evidence-based $\operatorname{care}^{55}$ ), as long as it is safe and does not extend treatment time to beyond 1 hour. We should also consider fidelity assessment of the interventions provided by the therapist to be certain that per-protocol treatment is being provided in each treatment arm.

A final modification would be the addition of economic analysis. Nepalese individuals are often poor, and the Nepalese public health system is resource poor. Not surprisingly, cost was a barrier to participation for $9 \%$ of potential participants. Pain education intervention appears to be a less resource-intensive alternative to current practice and could be delivered outside of the public health system, in community settings, although the costs and time of physiotherapist would be no different from guideline-based care as delivered in a physiotherapy department. Pain education might require more training of therapists, although training in guideline-based care may be necessary too. As such, a full trial would benefit from the addition of a full economic evaluation.

\section{Strengths and limitations}

The current study has a number of strengths: we used an active guideline-based care as the comparator group; we successfully blinded the assessor and analyst and assessed both blinding and contamination; outcomes were consistent with NIH recommendations on research standards for chronic LBP $^{38}$ and core outcome sets for LBP research ${ }^{39}$; and we submitted our protocol prior to data collection and remained transparent in all reporting. ${ }^{56}$

To our knowledge, this is the first study to examine the feasibility of a clinical trial on LBP in Nepal. Conducting a feasibility study is an important step before conducting a full clinical trial, ${ }^{47}$ especially in a setting where a highquality clinical trial has never been conducted, which lacks recommendations from previous experiences for such a study. For example, we had planned a full clinical trial in $2015^{57}$ but were unable to recruit participants because the clinicians were too busy to collect data and provide interventions as per protocol, and we encountered difficulty ensuring access to an assessor blinded to group allocation because of multiple responsibilities of the clinicians. These are feasibility problems that would have been revealed in a preliminary feasibility study. ${ }^{47}$

The current study also has a number of important limitations. Our follow-up was shorter than we would use in a full clinical trial. The short follow-up duration was chosen because 1-week assessment was sufficient to answer the feasibility-related questions, but whether longterm follow-ups are feasible in this setting and population remains to be demonstrated. We did not assess treatment fidelity in the current study, because we did not have the resources to do so. That the current practice in the LBP management at the study site was very different from clinical practice guidelines made it harder for the physiotherapists to comply with the guideline-based care. Another limitation was that we did not include any measure of physical activity as a secondary outcome, despite improved physical activity being one aim of pain education. In a definitive trial, we may consider using a measure to assess physical activity such as International Physical Activity Questionnaire, ${ }^{58}$ or an objective measure of physical activity, such as Actigraphy. ${ }^{59}$ Finally, the experimental group treatment was provided by the primary author of the study who may have inadvertently communicated more enthusiasm for the experimental group treatment than the therapists providing the CG treatment; this may have influenced the study findings. A full clinical trial would ideally include a number of therapists who would be trained to deliver both treatments, as one way to control for the therapist effects. This could also improve the generalisability of the study findings.

\section{SUMMARY AND CONCLUSIONS}

We conclude that a clinical trial to evaluate the effectiveness of pain education and evidence-based physiotherapy treatment in Nepal is feasible and warranted, although some minor modifications are required.

\section{Author affiliations}

${ }^{1}$ Department of Physiotherapy, Kathmandu University School of Medical Sciences, Dhulikhel, Bagmati, Nepal

${ }^{2}$ Centre for Musculoskeletal Outcomes Research, Department of Surgical Sciences, Dunedin School of Medicine, University of Otago, Dunedin, Otago, New Zealand ${ }^{3}$ Department of Rehabilitation Medicine, University of Washington, Seattle, Washington, USA 
${ }^{4}$ University of South Australia, Adelaide, South Australia, Australia

Acknowledgements Authors would like to thank Sahara Physiotherapy Hospital for providing permission to conduct the research and providing access to hospital space and patients. We are also thankful to the physiotherapists involved in the treatment of patients in the control group and the assessor (Bandana Gautam) for assisting with data collection. Finally, we would like to thank all the persons reviewing and providing valuable comments on the Nepali pain education handbook, especially Anupa Pathak, who provided feedback at all stages of patient handbook development.

Contributors SS: conception, design, development of pain education package in Nepali, data analysis and interpretation, drafting the manuscript, final approval of the manuscript. JHA: conception, design, interpretation, revision of the manuscript and final approval. MPJ: conception, design, revision of the manuscript and final approval. GLM: contributions to the development of pain education, study design, revision of the manuscript and final approval.

Funding The authors have not declared a specific grant for this research from any funding agency in the public, commercial or not-for-profit sectors. The study was supported by University of Otago Doctoral Scholarship for SS.

Competing interests GLM has received support from Pfizer, AIA Australia, Gallagher Bassett, Kaiser Permanente USA, Port Adelaide Football Club, Arsenal Football Club and the International Olympic Committee. GLM receives royalties for books on pain and rehabilitation, including the text on which the content for the proposed intervention was based. He also receives speaker fees for lectures on pain and rehabilitation. Neither GLM, nor the publishers of the Explain Pain materials, had any role in data collection or analysis or the decision to publish the data.

\section{Patient consent for publication Not required}

Ethics approval We obtained ethical approval from Nepal Health Research Council (reg. 422/2017) and the University of Otago Human Ethics Committee for Health (reg. H17/157).

Provenance and peer review Not commissioned; externally peer reviewed.

Data sharing statement Data are available from the principal investigator on request.

Open access This is an open access article distributed in accordance with the Creative Commons Attribution Non Commercial (CC BY-NC 4.0) license, which permits others to distribute, remix, adapt, build upon this work non-commercially, and license their derivative works on different terms, provided the original work is properly cited, appropriate credit is given, any changes made indicated, and the use is non-commercial. See: http://creativecommons.org/licenses/by-nc/4.0/.

\section{REFERENCES}

1. GBD 2016 Disease and Injury Incidence and Prevalence Collaborators. Global, regional, and national incidence, prevalence, and years lived with disability for 328 diseases and injuries for 195 countries, 1990-2016: a systematic analysis for the Global Burden of Disease Study 2016. Lancet 2017;390:1211-59.

2. Hartvigsen J, Hancock MJ, Kongsted A, et al. What low back pain is and why we need to pay attention. Lancet 2018;391:2356-67.

3. Buchbinder R, van Tulder M, Öberg B, et al. Low back pain: a call for action. Lancet 2018;391:2384-8.

4. Sharma S, Shrestha N, Jensen MP. Pain-related factors associated with lost work days in nurses with low back pain: a cross-sectional study. Scand J Pain 2016;11:27-33.

5. Paudyal P, Ayres JG, Semple S, et al. Low back pain among textile workers: a cross-sectional study. Occup Med 2013;63:129-34.

6. Qaseem A, Wilt TJ, McLean RM, et al. Noninvasive treatments for acute, subacute, and chronic low back pain: a clinical practice guideline from the american college of physicians. Ann Intern Med 2017;166:514-30.

7. Van Wambeke P, Desomer A, Ailiet L, et al. Low back pain and radicular pain: assessment and management. KCE Report 2017:287.

8. Almeida M, Saragiotto B, Richards B, et al. Primary care management of non-specific low back pain: key messages from recent clinical guidelines. Med J Aust 2018;208:272-5.

9. Foster NE, Anema JR, Cherkin D, et al. Prevention and treatment of low back pain: evidence, challenges, and promising directions. Lancet 2018;391:2368-83.

10. Maher C, Underwood M, Buchbinder R. Non-specific low back pain. Lancet 2017;389:736-47.
11. Bhattarai S, Chhetri HP, Alam K, et al. A study on factors affecting low back pain and safety and efficacy of NSAIDs in acute low back pain in a tertiary care hospital of Western Nepal. J Clin Diagn Res 2013;7:2752-8.

12. Bajracharya $S$, Singh M, Singh GK, et al. Clinico-epidemiological study of spinal injuries in a predominantly rural population of eastern Nepal: A 10 years' analysis. Indian J Orthop 2007;41:286-9.

13. Moseley GL. Whole of community pain education for back pain. Why does first-line care get almost no attention and what exactly are we waiting for? Br J Sports Med 2018:bjsports-2018-099567.

14. Moseley GL, Butler DS. Fifteen years of explaining pain: the past, present, and future. J Pain 2015;16:807-13.

15. Moseley GL, Nicholas MK, Hodges PW. A randomized controlled trial of intensive neurophysiology education in chronic low back pain. Clin $J$ Pain 2004;20:324-30.

16. Butler DS, Moseley GL. Explain pain. 2nd edn: Noigroup Publications, 2013.

17. Malfliet A, Kregel J, Coppieters I, et al. Effect of pain neuroscience education combined with cognition-targeted motor control training on chronic spinal pain: a randomized clinical trial. JAMA Neurol 2018;75:808

18. Louw A, Butler DS, Diener I, et al. Development of a preoperative neuroscience educational program for patients with lumbar radiculopathy. Am J Phys Med Rehabil 2013;92:446-52.

19. Louw A, Diener I, Landers MR, et al. Three-year follow-up of a randomized controlled trial comparing preoperative neuroscience education for patients undergoing surgery for lumbar radiculopathy. $J$ Spine Surg 2016;2:289-98.

20. Nijs J, Meeus M, Cagnie B, et al. A modern neuroscience approach to chronic spinal pain: combining pain neuroscience education with cognition-targeted motor control training. Phys Ther 2014;94:730-8.

21. Rizzo RRN, Medeiros FC, Pires LG, et al. Hypnosis enhances the effects of pain education in patients with chronic nonspecific low back pain: a randomized controlled trial. J Pain 2018;19:1103. e1-1103.e9.

22. Robins H, Perron V, Heathcote LC, et al. Pain neuroscience education: state of the art and application in pediatrics. Children 2016;3:43.

23. van Ittersum $M W$, van Wilgen $C P$, van der Schans $C P$, et al. Written pain neuroscience education in fibromyalgia: a multicenter randomized controlled trial. Pain Pract 2014;14:689-700.

24. Werner EL, Storheim K, Løchting I, et al. Cognitive patient education for low back pain in primary care: a cluster randomized controlled trial and cost-effectiveness analysis. Spine 2016;41:455-62.

25. Traeger $A C$, Lee $H$, Hübscher $M$, et al. Effect of intensive patient education vs placebo patient education on outcomes in patients with acute low back pain: a randomized clinical trial. JAMA Neurol 2018.

26. Gallagher L, McAuley J, Moseley GL. A randomized-controlled trial of using a book of metaphors to reconceptualize pain and decrease catastrophizing in people with chronic pain. Clin J Pain 2013;29:20-5.

27. Moseley GL. Evidence for a direct relationship between cognitive and physical change during an education intervention in people with chronic low back pain. Eur J Pain 2004;8:39-45.

28. Moseley G, Butler D. Explain pain supercharged. Adelaide, Australia: Noigroup publications, 2017.

29. Eldridge SM, Lancaster GA, Campbell MJ, et al. Defining feasibility and pilot studies in preparation for randomised controlled trials: development of a conceptual framework. PLoS One 2016;11:e0150205.

30. Moseley GL, Butler DSB. Explain pain handbook: Protectometer, 2015.

31. Sharma S, Jensen MP, Moseley GL, et al. Pain education for patients with non-specific low back pain in Nepal: protocol of a feasibility randomised clinical trial (PEN-LBP Trial). BMJ Open 2018;8:e022423.

32. Chan AW, Tetzlaff JM, Gøtzsche PC, et al. SPIRIT 2013 explanation and elaboration: guidance for protocols of clinical trials. BMJ 2013;346:e7586.

33. Eldridge SM, Chan CL, Campbell MJ, et al. CONSORT 2010 statement: extension to randomised pilot and feasibility trials. BMJ 2016;355:i5239.

34. Yamato T, Maher C, Saragiotto B, et al. The TIDieR checklist will benefit the physical therapy profession. J Orthop Sports Phys Ther 2016;46:402-4.

35. Hoffmann TC, Glasziou PP, Boutron I, et al. Better reporting of interventions: template for intervention description and replication (TIDieR) checklist and guide. BMJ 2014;348:g1687.

36. National Institute for Health and Care Excellence. Low back pain and sciatica in over 16s: assessment and management. 2016 https:// www.nice.org.uk/guidance/ng59 
37. Toward Optimized Practice (TOP) Low Back Pain Working Group. Evidence-informed primary care management of low back pain: clinical practice guideline. 2015 http://www.topalbertadoctors.org/ cpgs/885801

38. Deyo RA, Dworkin SF, Amtmann D, et al. Report of the nih task force on research standards for chronic low back pain. $J$ Pain 2014:15:569-85.

39. Chiarotto A, Boers M, Deyo RA, et al. Core outcome measurement instruments for clinical trials in nonspecific low back pain. Pain 2018:159:1-95

40. Deyo RA, Battie M, Beurskens AJ, et al. Outcome measures for low back pain research. A proposal for standardized use. Spine 1998:23:2003-13.

41. Sharma S, Pathak A, Maharjan R, et al. Psychometric properties of nepali versions of PROMIS short from measures of pain intensity, pain interference, pain behaviour, depressions, and sleep disturbance. The Journal of Pain 2018;19:S59.

42. Sharma S, Palanchoke J, Abbott JH. Cross-cultural adaptation and validation of the nepali translation of the patient-specific functional scale. J Orthop Sports Phys Ther 2018;48:1-23.

43. Sharma S, Palanchoke J, Reed D, et al. Translation, cross-cultural adaptation and psychometric properties of the Nepali versions of numerical pain rating scale and global rating of change. Health Qual Life Outcomes 2017;15:236.

44. Sharma S, Thibault P, Abbott JH, et al. Clinimetric properties of the nepali version of the pain catastrophizing scale in individuals with chronic pain. J Pain Res 2018;11:265-76.

45. Sharma S, Pathak A, Abbott JH, et al. Measurement properties of the Nepali version of the Connor Davidson resilience scales in individuals with chronic pain. Health Qual Life Outcomes 2018;16:56.

46. Billingham SAM, Whitehead AL, Julious SA. An audit of sample sizes for pilot and feasibility trials being undertaken in the United Kingdom registered in the United Kingdom clinical research network database. BMC Med Res Methodol 2013:13:104.

47. Abbott $\mathrm{JH}$. The distinction between randomized clinical trials (RCTs) and preliminary feasibility and pilot studies: what they are and are not. J Orthop Sports Phys Ther 2014;44:555-8.

48. Dettori JR. Loss to follow-up. Evid Based Spine Care J 2011;2:7-10.

49. Greenhalgh T, Hurwitz B. Why study narrative? BMJ 1999;318:48-50.

50. Richards T. Listen to patients first. BMJ 2014;349:95765.

51. Block AR, Kremer EF, Gaylor M. Behavioral treatment of chronic pain: the spouse as a discriminative cue for pain behavior. Pain 1980;9:243-52.

52. Flor H, Kerns RD, Turk DC. The role of spouse reinforcement, perceived pain, and activity levels of chronic pain patients. $J$ Psychosom Res 1987;31:251-9.

53. Fergusson D, Glass KC, Waring D, et al. Turning a blind eye: the success of blinding reported in a random sample of randomised, placebo controlled trials. BMJ 2004;328:432.

54. Schulz KF, Grimes DA. Blinding in randomised trials: hiding who got what. The Lancet 2002;359:696-700.

55. Sackett DL, Rosenberg WM, Gray JA, et al. Evidence based medicine: what it is and what it isn't. BMJ 1996;312:71-2.

56. Lee H, Lamb SE, Bagg MK, et al. Reproducible and replicable pain research: a critical review. Pain 2018;159:1683-1689.

57. ClinicalTrials.gov. Identifier: NCT02343315. Are Supervised Exercises and TENS necessary along with prescribed home exercises for chronic low back pain? Bethesda (MD): National Library of Medicine (US), 2015.

58. Craig CL, Marshall AL, Sjöström M, et al. International physical activity questionnaire: 12-country reliability and validity. Med Sci Sports Exerc 2003;35:1381-95.

59. Patterson SM, Krantz DS, Montgomery LC, et al. Automated physical activity monitoring: validation and comparison with physiological and self-report measures. Psychophysiology 1993;30:296-305. 